\title{
ON ORDERS OF OPTIMAL NORMAL BASIS GENERATORS
}

\author{
SHUHONG GAO AND SCOTT A. VANSTONE
}

\begin{abstract}
In this paper we give some experimental results on the multiplicative orders of optimal normal basis generators in $F_{2^{n}}$ over $F_{2}$ for $n \leq 1200$ whenever the complete factorization of $2^{n}-1$ is known. Our results show that a subclass of optimal normal basis generators always have high multiplicative orders, at least $O\left(\left(2^{n}-1\right) / n\right)$, and are very often primitive. For a given optimal normal basis generator $\alpha$ in $F_{2^{n}}$ and an arbitrary integer $e$, we show that $\alpha^{e}$ can be computed in $O(n \cdot v(e))$ bit operations, where $v(e)$ is the number of 1 's in the binary representation of $e$.
\end{abstract}

For a prime power $q$ and a positive integer $n$, let $F_{q}$ and $F_{q^{n}}$ be the finite fields of $q$ and $q^{n}$ elements, respectively. A normal basis $N$ for $F_{q^{n}}$ over $F_{q}$ is a basis of the form $\left(\alpha, \alpha^{q}, \ldots, \alpha^{q^{n-1}}\right)$, where $\alpha \in F_{q^{n}}$. In this case $\alpha$ is said to be a normal element or normal basis generator. The complexity of $N$, denoted by $c_{N}$, is defined to be the number of nonzero entries $t_{i j}$ in the $n$ expressions

$$
\alpha \cdot \alpha^{q^{i}}=\sum_{j=0}^{n-1} t_{i j} \alpha^{q^{j}}, \quad 0 \leq i \leq n-1,
$$

where $t_{i j} \in F_{q}$. It is easy to prove that $c_{N} \geq 2 n-1$. If $c_{N}=2 n-1$, then $N$ is called an optimal normal basis. Mullin, Onyszchuk, Vanstone and Wilson [11] constructed the following two families of optimal normal bases, which are essentially all the optimal normal bases in finite fields as shown by Gao and Lenstra [6].

Construction I. Suppose $n+1$ is a prime and $q$ is primitive in $Z_{n+1}$, where $q$ is a prime or prime power. Then any primitive $(n+1)$ st root of unity generates an optimal normal basis for $F_{q^{n}}$ over $F_{q}$.

Construction II. Let $2 n+1$ be a prime and assume that $Z_{2 n+1}^{*}$ is generated by 2 and -1 . Then $\alpha=\gamma+\gamma^{-1}$ generates an optimal normal basis for $F_{2^{n}}$ over $F_{2}$, where $\gamma$ is a primitive $(2 n+1)$ st root of unity.

Optimal normal bases have been successfully used in hardware implementation of large finite fields in order to construct cryptosystems which are secure and efficient (see for example [1, 2]). There are several reasons for the interest in optimal normal bases, and in normal bases in general. When elements of

Received by the editor June 6, 1994.

1991 Mathematics Subject Classification. Primary 11T30; Secondary 11Y16, 12Y05, 68Q25.

Key words and phrases. Finite fields, primitive elements, normal bases.

The first author was supported by an NSERC post doctoral fellowship.

(C)1995 American Mathematical Society 
$F_{q^{n}}$ are represented under a normal basis over $F_{q}$, taking a $q$ th power of an element in $F_{q^{n}}$ is just a cyclic shift of coordinates, so the cost is almost negligible. In practice $q=2$, exponentiation by repeated square and multiply can be greatly expedited. Another nice property about normal bases is that their multiplication tables possess many symmetries, which is useful in practical implementation of finite fields. For optimal normal bases, they have the additional property of being self-dual (Construction II only) and having the lowest complexity, which makes it feasible for hardware realization of large finite fields.

In several cryptographic systems (including exponential pseudorandom number generators), a fixed element of a group needs to be repeatedly raised to many different large powers. To make such systems secure, the fixed element must have high order. In any implementation of these systems, there should be an efficient algorithm for computing large powers of the fixed element. In this paper, we show by experimental results that the optimal normal basis generators given in Construction II have exactly this desired property: they have very high multiplicative orders, and large powers of them can be computed efficiently, as indicated by the following result.

Theorem. Let $\alpha$ be as in Construction II. Then, for any integer $e, \alpha^{e}$ can be computed in $O(n \cdot v(e))$ bit operations, where $v(e)$ denotes the number of 1 's in the binary representation of $e$.

As $v(e) \leq n$ for $0 \leq e \leq 2^{n}-1, \alpha^{e}$ can be computed in $O\left(n^{2}\right)$ bit operations. In comparison, we should mention that for an arbitrary $\beta \in F_{2^{n}}$, if $F_{2^{n}}$ is represented by an optimal normal basis, Stinson [13] and von zur Gathen [7] showed that $\beta^{e}$ can be computed in about $O\left(n / \log _{2} n\right)$ multiplications in $F_{2^{n}}$, and thus in $O\left(n^{3} / \log _{2} n\right)$ bit operations, where squaring is considered free and one multiplication in $F_{2^{n}}$ under the normal basis needs $O\left(n^{2}\right)$ bit operations. If $F_{2^{n}}$ is represented by a power basis (which is of the form $1, \xi, \ldots, \xi^{n-1}$ ), then, by using fast algorithms $[12,5]$ for multiplication, $\beta^{e}$ can be computed by the square and multiply method in $O(n \log n \log \log n \log e)$, or $O\left(n^{2} \log n \log \log n\right)$ bit operations. It is not known how to improve the time $O(n \cdot v(e))$ with precomputations as in [3]. The reason is that we do not have an $O(n)$ algorithm for computing the product of two arbitrary elements in $F_{2^{n}}$.

In the following, we assume that the conditions in Construction II are satisfied. Our goal is to determine the multiplicative order of $\alpha=\gamma+\gamma^{-1}$. We will compute in $F_{2^{n}}$ represented under the optimal normal basis generated by $\alpha$ with basis elements ordered differently.

We use the standard algorithm in [9, page 87] for determining the multiplicative orders of elements in finite fields. To apply this algorithm for computing the multiplicative order of an element in $F_{2^{n}}$, one needs to know the complete factorization of the integer $2^{n}-1$. Tables of factorizations of integers of the form $b^{n} \pm 1$ for small $b$ and $n$ are given in [4] and updated versions are available from the authors. In the following, we show how to efficiently compute $\alpha^{e}$ for an arbitrary integer $e$.

The optimal normal basis generated by $\alpha$ is $\left(\alpha, \alpha^{2}, \ldots, \alpha^{2^{n-1}}\right)$. We will arrange the elements of the basis in a different order. For an integer $i$, define $\gamma_{i}=\gamma^{i}+\gamma^{-i}$. Obviously, $\gamma_{0}=0$ and $\gamma_{1}=\alpha$. As the multiplicative order of $\gamma$ is $2 n+1$, it is easy to check that $\gamma_{i}=\gamma_{j}$ if and only if $i \equiv \pm j \bmod 2 n+1$. 
So $\gamma_{1}, \gamma_{2}, \ldots, \gamma_{n}$ are all the distinct nonzero $\gamma_{i}$ 's. We claim that

$$
\left\{\alpha, \alpha^{2}, \ldots, \alpha^{2^{n-1}}\right\}=\left\{\gamma_{1}, \gamma_{2}, \ldots, \gamma_{n}\right\}
$$

The reason is that for each $0 \leq i \leq n-1, \alpha^{2^{i}}=\gamma^{2^{i}}+\gamma^{-2^{i}}=\gamma_{2^{i}}$ belongs to the set of the right-hand side, while for each $1 \leq i \leq n$, since $Z_{2 n+1}^{*}$ is generated by 2 and -1 , there is an integer $k$ such that $i \equiv \pm 2^{k} \bmod 2 n+1$, and thus $\gamma_{i}=\alpha^{2^{k}}$ belongs to the set of the left-hand side.

Therefore, $\gamma_{1}, \gamma_{2}, \ldots, \gamma_{n}$ form a basis of $F_{2^{n}}$ over $F_{2}$. To facilitate multiplication of elements represented under this basis, we define a new function from the set of integers to the set $\{0,1, \ldots, n\}$. For any integer $i$, define $s(i)$ to be the unique integer such that

$$
0 \leq s(i) \leq n, \quad \text { and } i \equiv s(i) \bmod 2 n+1 \text { or } i \equiv-s(i) \bmod 2 n+1 .
$$

Obviously, $s(0)=0, s(i)=s(-i)$ and

$$
\gamma_{i}=\gamma_{s(i)}, \quad \alpha^{2^{i}}=\gamma_{s\left(2^{i}\right)} \text { for all } i
$$

As $\gamma_{i} \cdot \gamma_{j}=\gamma_{i+j}+\gamma_{i-j}$ for all $i, j$, we have

$$
\gamma_{i} \cdot \gamma_{j}=\gamma_{s(i+j)}+\gamma_{s(i-j)}, \quad 1 \leq i, j \leq n \text {. }
$$

Next we show how to compute the product $\gamma_{i} \cdot A$, where $1 \leq i \leq n$ and $A$ is an arbitrary element in $F_{2^{n}}$. Suppose that $A=\sum_{k=1}^{n} a_{k} \gamma_{k}$, where $a_{k} \in F_{2}$. Then

$$
\gamma_{i} \cdot A=\sum_{k=1}^{n} a_{k} \gamma_{i} \cdot \gamma_{k}=\sum_{k=1}^{n} a_{k}\left(\gamma_{s(k+i)}+\gamma_{s(k-i)}\right)
$$

Note that

$$
\begin{aligned}
\sum_{k=1}^{n} a_{k} \gamma_{s(k+i)} & =\sum_{k=1}^{n-i} a_{k} \gamma_{k+i}+\sum_{k=n+1-i}^{n} a_{k} \gamma_{2 n+1-(k+i)} \\
& =\sum_{k=i+1}^{n} a_{k-i} \gamma_{k}+\sum_{k=n+1-i}^{n} a_{2 n+1-(k+i)} \gamma_{k} \\
& =\sum_{k=i+1}^{n} a_{s(k-i)} \gamma_{k}+\sum_{k=n+1-i}^{n} a_{s(k+i)} \gamma_{k}, \\
\sum_{k=1}^{n} a_{k} \gamma_{s(k-i)} & =\sum_{k=1}^{i} a_{k} \gamma_{i-k}+\sum_{k=i+1}^{n} a_{k} \gamma_{k-i} \\
& =\sum_{k=1}^{i} a_{i-k} \gamma_{k}+\sum_{k=1}^{n-i} a_{k+i} \gamma_{k} \\
& =\sum_{k=1}^{i} a_{s(k-i)} \gamma_{k}+\sum_{k=1}^{n-i} a_{s(k+i)} \gamma_{k},
\end{aligned}
$$


where here, and hereafter, we assume that $a_{0}=0$. We see that

$$
\begin{aligned}
\gamma_{i} \cdot A & =\sum_{k=1}^{n}\left(a_{s(k-i)}+a_{s(k+i)}\right) \gamma_{k} \\
& =\sum_{k=1}^{c}\left(a_{i-k}+a_{k+i}\right) \gamma_{k}+\sum_{k=c+1}^{d} f(k) \gamma_{k}+\sum_{k=d+1}^{n}\left(a_{k-i}+a_{2 n+1-(k+i)}\right) \gamma_{k},
\end{aligned}
$$

where $c=\min \{i, n-i\}, d=\max \{i, n-i\}=n-c$ and

$$
f(k)= \begin{cases}a_{i-k}+a_{2 n+1-(k+i)} & \text { if } i>n-i, \\ a_{k-i}+a_{k+i} & \text { if } i<n-i .\end{cases}
$$

This shows that $\gamma_{i} \cdot A$ can be computed in $O(n)$ bit operations.

Now, to compute $\alpha^{e}$ we can assume that $0 \leq e<2^{n}-1$, as $\alpha^{2^{n}-1}=1$. Write $e=\sum_{k=0}^{n-1} e_{k} 2^{k}$, where $e_{k} \in\{0,1\}$. Then

$$
\alpha^{e}=\prod_{k=0}^{n-1}\left(\alpha^{2^{k}}\right)^{e_{k}}=\prod_{k=0}^{n-1}\left(\gamma_{s\left(2^{k}\right)}\right)^{e_{k}} .
$$

This suggests that $\alpha^{e}$ can be computed iteratively as follows:

Algorithm:

Input: An integer $e$ with $0 \leq e \leq 2^{n}-1$.

Output: $\alpha^{e}$ represented in the basis $\left(\gamma_{1}, \ldots, \gamma_{n}\right)$.

Step 1. Set $A:=1=\sum_{k=1}^{n} \gamma_{k}$ and compute the binary representation:

$$
e=\sum_{k=0}^{n-1} e_{k} 2^{k}
$$

Step 2. For $k$ from 0 to $n-1$, if $e_{k}=1$ then set $A:=\gamma_{s\left(2^{k}\right)} \cdot A$;

Step 3. Return $A$;

End.

The correctness of the algorithm is obvious. The major cost is incurred at Step 2 where $v(e)$ products of the form $\gamma_{k} \cdot A$ are computed. Since we have shown that each such product can be computed in $O(n)$ bit operations, the total cost is $O(n \cdot v(e))$ bit operations. Therefore, $\alpha^{e}$ can be computed in $O(n \cdot v(e))$ bit operations, as claimed by the theorem above.

By using the algorithms described above, we have computed the multiplicative order of $\alpha$ for $n \leq 1200$ where the conditions of Construction II are satisfied and the complete factorization of $2^{n}-1$ is known. The results are summarized in Table 1 . The index of $\alpha$ in $F_{2^{n}}$ is defined to be $\left(2^{n}-1\right) / e$, where $e$ is the multiplicative order of $\alpha$. Thus, index 1 in the table means that the corresponding $\alpha$ is a primitive element. The "?" in the table means that the complete factorization of the corresponding number $2^{n}-1$ is not known yet, and thus the index computed from the partial factorization may not be the true index.

Table 1 indicates that the multiplicative order of $\alpha$ is at least $O\left(\left(2^{n}-1\right) / n\right)$. This means that $\alpha$ always has very high multiplicative order. The last two values of $n$ in the table are Mersenne primes; the corresponding optimal normal basis generators $\alpha$ are automatically primitive. Note that $\alpha$ is frequently primitive. In particular, one can check that if $n$ is prime, then $\alpha$ is primitive in the table. We conjecture that this is always so, as stated below. 
TABLE 1. Indices of optimal normal basis generators in $F_{2^{n}}$

\begin{tabular}{|c|c|c|c|c|c|c|c|c|c|}
\hline$n$ & Index & $n$ & Index & $n$ & Index & $n$ & Index & $n$ & Index \\
\hline 2 & 1 & 183 & 1 & 413 & 1 & 683 & $1 ?$ & 950 & 3 \\
\hline 3 & 1 & 186 & 3 & 414 & 3 & 686 & 3 & 953 & $1 ?$ \\
\hline 5 & 1 & 189 & 1 & 419 & 1 & 690 & 151 & 965 & 1 \\
\hline 6 & 1 & 191 & 1 & 426 & 1 & 713 & $1 ?$ & 974 & 3 \\
\hline 9 & 1 & 194 & 3 & 429 & 1 & 719 & $1 ?$ & 975 & 1 \\
\hline 11 & 1 & 209 & 1 & 431 & 1 & 723 & 1 & 986 & 3 \\
\hline 14 & 1 & 210 & 1 & 438 & 3 & 725 & $1 ?$ & 989 & $1 ?$ \\
\hline 18 & 3 & 221 & 1 & 441 & 1 & 726 & 1 & 993 & 1 \\
\hline 23 & 1 & 230 & 1 & 443 & 1 & 741 & 7 & 998 & 1 \\
\hline 26 & 1 & 231 & 1 & 453 & 1 & 743 & $1 ?$ & 1013 & $1 ?$ \\
\hline 29 & 1 & 233 & 1 & 470 & 1 & 746 & 1 & 1014 & 7 \\
\hline 30 & 1 & 239 & 1 & 473 & 1 & 749 & $1 ?$ & 1019 & $1 ?$ \\
\hline 33 & 1 & 243 & 1 & 483 & 1 & 755 & 1 & 1026 & 7 \\
\hline 35 & 1 & 245 & 1 & 491 & 1 & 761 & $1 ?$ & 1031 & $1 ?$ \\
\hline 39 & 1 & 251 & 1 & 495 & 1 & 765 & 1 & 1034 & 3 \\
\hline 41 & 1 & 254 & 1 & 509 & 1 & 771 & 1 & 1041 & 1 \\
\hline 50 & 3 & 261 & 1 & 515 & 1 & 774 & 1 & 1043 & $1 ?$ \\
\hline 51 & 1 & 270 & 7 & 519 & 1 & 779 & $1 ?$ & 1049 & 1 \\
\hline 53 & 1 & 273 & 1 & 530 & 1 & 783 & $7 ?$ & 1055 & $1 ?$ \\
\hline 65 & 1 & 278 & 3 & 531 & 1 & 785 & $1 ?$ & 1065 & $1 ?$ \\
\hline 69 & 1 & 281 & 1 & 543 & 1 & 791 & $1 ?$ & 1070 & 1 \\
\hline 74 & 1 & 293 & 1 & 545 & 1 & 803 & $23 ?$ & 1103 & 1 \\
\hline 81 & 1 & 299 & 1 & 554 & 1 & 809 & $1 ?$ & 1106 & 381 \\
\hline 83 & 1 & 303 & 1 & 558 & 1 & 810 & 1 & 1110 & 9 \\
\hline 86 & 1 & 306 & 1 & 561 & 1 & 818 & 1 & 1118 & $1 ?$ \\
\hline 89 & 1 & 309 & 1 & 575 & 1 & 831 & 1 & 1119 & 1 \\
\hline 90 & 1 & 323 & 1 & 585 & 1 & 833 & $1 ?$ & 1121 & 1 \\
\hline 95 & 1 & 326 & 1 & 593 & $1 ?$ & 834 & 1 & 1133 & $1 ?$ \\
\hline 98 & 3 & 329 & 1 & 606 & 9 & 846 & 1 & 1134 & 3 \\
\hline 99 & 7 & 330 & 1 & 611 & $1 ?$ & 866 & 1 & 1146 & 1 \\
\hline 105 & 1 & 338 & 3 & 614 & 3 & 870 & 1 & 1154 & 1 \\
\hline 113 & 1 & 350 & 3 & 615 & 1 & 873 & 1 & 1155 & 1 \\
\hline 119 & 1 & 354 & 3 & 618 & 1 & 879 & 1 & 1166 & 1 \\
\hline 131 & 1 & 359 & 1 & 629 & $1 ?$ & 891 & 1 & 1169 & 1 \\
\hline 134 & 3 & 371 & 1 & 638 & 1 & 893 & $1 ?$ & 1178 & $3 ?$ \\
\hline 135 & 1 & 375 & 1 & 639 & 1 & 911 & $1 ?$ & 1185 & 1 \\
\hline 146 & 1 & 378 & 3 & 641 & $1 ?$ & 923 & $1 ?$ & 1194 & $1 ?$ \\
\hline 155 & 1 & 386 & 1 & 645 & 7 & 930 & 3 & 1199 & $1 ?$ \\
\hline 158 & 1 & 393 & 7 & 650 & 3 & 933 & $1 ?$ & 1211 & $1 ?$ \\
\hline 173 & 1 & 398 & 1 & 651 & 1 & 935 & $1 ?$ & 1218 & $1 ?$ \\
\hline 174 & 3 & 410 & 11 & 653 & 1 & 938 & 1 & 9689 & 1 \\
\hline 179 & 1 & 411 & 1 & 659 & $1 ?$ & 939 & $1 ?$ & 21701 & 1 \\
\hline
\end{tabular}


TABLE 2. Cunningham chains of primes

\begin{tabular}{|r|r|r|r|r|}
\hline 1122659 & 2164229 & 2329469 & 10257809 & 10309889 \\
2245319 & 4328459 & 4658939 & 20515619 & 20619779 \\
4490639 & 8656919 & 9317879 & 41031239 & 41239559 \\
8981279 & 17313839 & 18635759 & 82062479 & 82479119 \\
17962559 & 34627679 & 37271519 & 164124959 & 164958239 \\
35925119 & 69255359 & 74543039 & 328249919 & 329916479 \\
71850239 & 138510719 & 149086079 & 656499839 & 659832959 \\
\hline
\end{tabular}

Conjecture. Suppose that $n$ and $2 n+1$ are both primes. Then, for any primitive $(2 n+1)$ st root of unity $\gamma$ in $F_{2^{2 n}}, \alpha=\gamma+\gamma^{-1}$ is a primitive element in $F_{2^{n}}$.

Note that when $n$ and $2 n+1$ are both primes, $Z_{2 n+1}^{*}$ is always generated by 2 and -1 . So the conditions in Construction II are satisfied and $\alpha$ generates an optimal normal basis for $F_{2^{n}}$ over $F_{2}$, which is easily seen to be a selfdual basis. This means that if the conjecture is true, then $\alpha$ generates a selfdual, primitive optimal normal basis for $F_{2^{n}}$ over $F_{2}$. Also note that it is not known if there are infinitely many integers $n$ such that both $n$ and $2 n+1$ are primes, though it is conjectured so. It is interesting to note that D.H. Lehmer [8] found several chains of primes with each member one more than twice the previous one (such chains are called Cunningham chains), as listed in columns of Table 2.

Finally, we remark that the inverse of $\alpha$ is easy to compute. Actually, if $\alpha^{-1}=\sum_{k=1}^{n} a_{k} \gamma_{k}$, then

$$
a_{k}=1 \text { if } \ell \text { is odd, and } 0 \text { if } \ell \text { is even, }
$$

where $\ell=k / 2$ if $k$ is even and $\ell=n-(k-1) / 2$ if $k$ is odd. So $\alpha^{-1}$ can be computed in $O(n)$ bit operations.

\section{ACKNOWLEDGMENT}

The authors would like to thank Joachim von zur Gathen for his helpful comments on an earlier draft of the paper. Thanks also go to all the people who contributed to the Cunningham project [4]. In particular, we would like to thank S.S. Wagstaff, Jr. for collecting the new factors and making them electronically available. Also we used MAPLE in our computing. Part of Table 1 has appeared in [10].

\section{BIBLIOGRAPHY}

1. G. B. Agnew, R. C. Mullin, I. M. Onyszchuk, and S. A. Vanstone, An implementation for a fast public key cryptosystem, J. Cryptology 3 (1991), 63-79.

2. G. B. Agnew, R. C. Mullin, and S. A. Vanstone, An implementation of elliptic curve cryptosystems over $F_{2155}$, IEEE J. on Selected Areas in Communications 11 (1993), 804813.

3. E. F. Brickell, D. M. Gordon, K. S. McCurley, and D. B. Wilson, Fast exponentiation with precomputation, Proc. Eurocrypt'92, Balatonfured, Hungary, 1992.

4. J. Brillhart, D. H. Lehmer, J. L. Selfridge, B. Tuckerman, and S. S. Wagstaff, Jr., Factorizations of $b^{n} \pm 1, b=2,3,5,6,7,10,11,12$ up to high powers, 2nd ed., Contemp. Math., vol. 22, Amer. Math. Soc., Providence, RI, 1988. 
5. D. G. Cantor and E. Kaltofen, On fast multiplication of polynomials over arbitrary algebras, Acta Inform. 28 (1991), 693-701.

6. S. Gao and H. W. Lenstra, Jr., Optimal normal bases, Des. Codes Cryptogr. 2 (1992), 315 323.

7. J. von zur Gathen, Efficient and optimal exponentiation in finite fields, Comput. Complexity 1 (1991), 360-394.

8. D. H. Lehmer, On certain chains of primes, Proc. London Math. Soc. 14A (Littlewood 80 volume, 1965), 183-186.

9. R. Lidl and H. Niederreiter, Finite fields, Addison-Wesley, Reading, MA, 1983. (Now distributed by Cambridge University Press.)

10. A. J. Menezes, I. F. Blake, X. Gao, R. C. Mullin, S. A. Vanstone, and T. Yaghoobian, Applications of finite fields, Kluwer, Boston-Dordrecht-Lancaster, 1993.

11. R. C. Mullin, I. M. Onyszchuk, S. A. Vanstone, and R. M. Wilson, Optimal normal bases in $G F\left(p^{n}\right)$, Discrete Appl. Math. 22 (1988/1989), 149-161.

12. A. Schönhage and V. Strassen, Schnelle Multiplikation großer Zahlen, Computing 7 (1971), 281-292.

13. D. R. Stinson, Some observations on parallel algorithms for fast exponentiation in $G F\left(2^{n}\right)$, SIAM J. Comput. 19 (1990), 711-717.

Department of Computer Science, University of Toronto, Toronto, Ontario, M5S 1A4, CANADA

E-mail address: sgao@cs.toronto.edu

Department of Combinatorics and Optimization, University of Waterloo, Waterloo, Ontario, N2L 3G1, Canada

E-mail address: savanstone@math. uwaterloo.ca 